\title{
Improvement of the convergence characteristics of the ANC system with the LMS algorithm by reducing the effect of secondary paths
}

\author{
Guoyue Chen, ${ }^{*}$ Masato Abe, ${ }^{* *}$ and Toshio Sone* \\ ${ }^{*}$ Research Institute of Electrical Communication/ \\ Graduate School of Information Sciences, Tohoku University, \\ 2-1-1, Katahira, Aoba-ku, Sendai, 980-77 Japan \\ **Computer Center, Tohoku University, \\ Aramaki, Aza Aoba, Aoba-ku, Sendai, 980-77 Japan
}

(Received 1 February 1996)

\begin{abstract}
The convergence characteristics of the feedforward active noise control system with the filtered-x LMS or multiple error filtered-x LMS algorithm are degraded by the transfer functions from the secondary sources to the error sensors (secondary paths). The possible methods to improve the convergence characteristics of the active noise control system with the LMS algorithm by reducing the effect of the secondary paths are discussed in this paper. In the first method, an index in the case of multiple secondary sources and error sensors to evaluate are introduced whether their positions are good or not before performing the cancellation. In the second method in which the number of secondary sources is increased, it is shown that the LMS adaptive algorithm performs well. In the third method with the use of the inverse filters of the secondary paths, the adaptive LMS algorithm is formulated and it is shown from the result of with a simulation using a transfer function measured in a room with a reverberation time of $0.5 \mathrm{~s}$ that this method is practically effective.
\end{abstract}

Keywords : Multiple error filtered-x LMS algorithm, Convergence characteristics, Secondary paths, Improvement, Matrix determinant

PACS number: 43. 50. Ki, 43. 50. Gf

\section{INTRODUCTION}

The filtered-x LMS algorithm ${ }^{1)}$ or the multiple error filtered-x (MEFX) LMS algorithm ${ }^{2}$ ) is widely used in feedforward active noise control (ANC) systems. The filtered-x LMS algorithm is an alternate form of the LMS algorithm, used when the secondary path from the adaptive filter output to the error sensor is represented by a non-unitary transfer function.

The convergence characteristics in ANC systems with these algorithms have been studied in the frequency domain in previous works. ${ }^{3,4)}$ The convergence characteristics can be evaluated in the frequency domain with a fewer computations and a better understanding of the physical meaning than in the time domain. The convergence characteristics with these algorithms are affected by the determinant of the matrices composed of the secondary paths (the transfer functions from the secondary sources to the error sensors) in the ANC system. The secondary paths are assumed to be known in this paper. At a frequency (FFT) bin where this determinant is relatively smaller, the convergence speed of the adaptive filter is slower, and it leads to a larger computation error and less cancellation. In other words, when the difference of the determinant is smaller throughout the frequency range concerned, the convergence speed is faster and the cancellation of noise is larger. 
In this paper, we discuss on the three possible methods to improve the convergence characteristics with the filtered-x LMS or the MEFX LMS algorithms, some of which were suggested or proposed, but they have not been investigated in detail. The first method is to adjust the positions of secondary sources and error sensors suggested by Stell and Bernhard. ${ }^{5)}$ In the case of a single secondary source and a single error sensor, whether their positions are good or not is easily evaluated by calculating the transfer function from the secondary source to the error sensor before performing the cancellation. ${ }^{3)}$ In the case of multiple secondary sources and multiple error sensors, however, such an effective evaluation method has not been reported. The second method is to increase the number of secondary sources showed by Miyoshi and Kaneda ${ }^{6)}$ when there is no common zero in the transfer functions of the secondary paths. However, whether the LMS adaptive algorithm works well or not has not been investigated. The third one is to increase the value of the determinant of the transfer function matrices composed of the secondary paths by using the inverse filters of the secondary paths. ${ }^{7}$ However, whether a higher convergence speed is practically achieved or not has not been investigated.

First this paper introduces an index to evaluate the convergence characteristics with the filtered-x LMS or the MEFX LMS algorithm, and shows that they are improving in the above mentioned three methods by making the determinant of the transfer function matrices as flat as possible over the relevant frequency range, and they can be practically applicable to the ANC system using the adaptive algo- rithms.

\section{THE EFFECTS OF SECONDARY PATHS ON THE CONVERGENCE CHARACTERISTICS ANALYZED IN THE FREQUENCY DOMAIN}

2.1 Review of the MEFX LMS Algorithm in the Time Domain

Figure 1 shows the general multiple channel ANC system. Here, $K$ is the number of reference sensors of the adaptive digital filter (ADF) array, composed of finite impulse response (FIR) filters, $M$ is the number of secondary sources, and $L$ is the number of error sensors (control points). Such a system will be referred to as CASE $[K, M, L]$ in this paper. There are $M \times L$ different secondary paths (secondary path matrix) between each secondary source and each error sensor, all of which have to be modeled and used $K$ times to generate the array of coefficients of each of the $K \times M$ adaptive filters in the controller, which drives every secondary source by every reference signal. In this paper, the multiple secondary paths are assumed to be time invariant and known.

Referring to Fig. 1, the MEFX LMS algorithm, which is an extension of the filtered-x LMS algorithm for the CASE $[K, M, L]$ ANC system, can be summarized as follows ${ }^{4,8)}$ :

$$
\begin{gathered}
\boldsymbol{e}(n)=\boldsymbol{d}(n)+\boldsymbol{U}(n) \boldsymbol{w}(n), \\
\boldsymbol{w}(n+1)=\boldsymbol{w}(n)-2 \mu \boldsymbol{U}^{\mathrm{T}}(n) \boldsymbol{e}(n),
\end{gathered}
$$

where the superscript $\mathrm{T}$ denotes the transpose of a matrix, $\mu$ is the step-size parameter, $\boldsymbol{U}(n)$ is a matrix

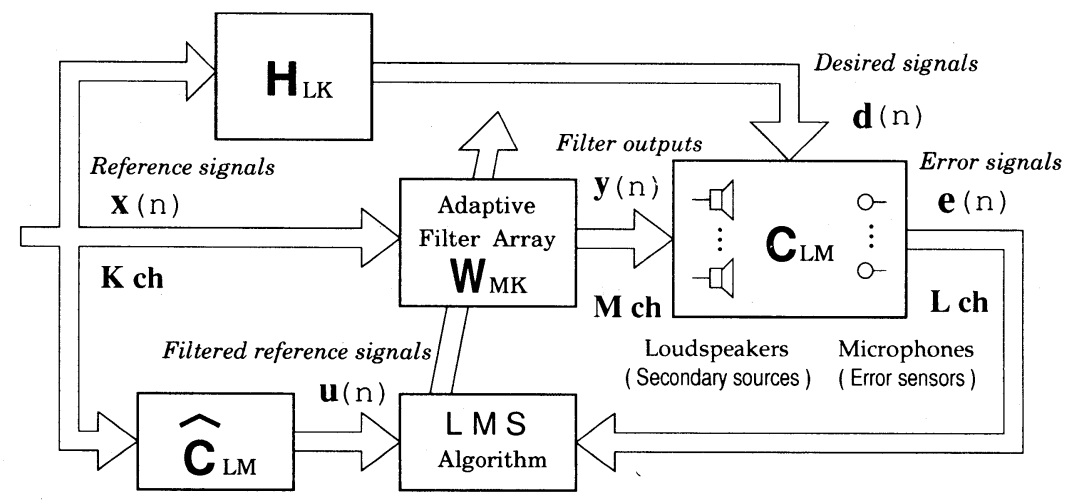

Fig. 1 Block diagram of the general multiple channel active noise control system, CASE $[K, M, L]$, using the multiple error filtered-x LMS algorithm. 


\section{G. CHEN et al.: IMPROVEMENT ANC SYSTEM CHARACTERISTICS}

of the filtered reference signal, and $e(n), d(n)$ and $\boldsymbol{w}(n)$ are the vectors of the error signals, desired signals and the adaptive filter weights, respectively.

The convergence speed with the MEFX LMS algorithm is subject to the distribution of eigenvalues of the autocorrelation matrix of the filtered reference signals, ${ }^{8)}$ as defined by

$$
\boldsymbol{R}=E\left[\boldsymbol{U}^{\mathrm{T}}(n) \boldsymbol{U}(n)\right],
$$

where $E[\quad]$ is the statistical expectation operator. Here, assuming that the reference signals are statistically stationary, time index $n$ of the matrix $\boldsymbol{R}$ is dropped.

The convergence characteristics with the MEFX LMS algorithm in the time domain are subject to the distribution of the eigenvalues of the autocorrelation matrix $\boldsymbol{R}$ of the filtered reference signal. However, the physical meaning, that is, the relation between the eigenvalue of the matrix $\boldsymbol{R}$ and the transfer function of the secondary path, is unclear, and it is not easy to find a method to improve the convergence characteristics.

\subsection{Review of Evaluation in the Frequency Domain}

When the convergence speed with the MEFX LMS algorithm is slow, the adaptive filters are considered as time invariant linear filters for a short period. Thus the MEFX LMS algorithm described in Eqs. (1) and (2) in the time domain can be approximately expressed in the frequency domain, ${ }^{4)}$ as in the following equations :

$$
\begin{aligned}
& \boldsymbol{E}(n, \omega)=\boldsymbol{D}(n, \omega)+\boldsymbol{U}(n, \omega) \boldsymbol{W}(n, \omega), \\
& \boldsymbol{W}(n+1, \omega) \\
& \quad=\boldsymbol{W}(n, \omega)-2 \mu^{\prime} \boldsymbol{U}^{\mathrm{H}}(n, \omega) \boldsymbol{E}(n, \omega),
\end{aligned}
$$

where $\mu^{\prime}=\mu / N(N$ is the length of FFT $)$, the superscript $\mathrm{H}$ denotes the Hermitian transpose, $\boldsymbol{E}(n, \omega)$, $\boldsymbol{D}(n, \omega)$ and $\boldsymbol{W}(n, \omega)$ are the FFT's of $\boldsymbol{e}(n), \boldsymbol{d}(n)$ and $\boldsymbol{w}(n)$, respectively, at the $n$-th iteration, and $\boldsymbol{U}(n, \omega)$ is a spectral matrix of the filtered reference signals. Equations (4) and (5) are valid independently at each frequency bin $\omega$ at the $n$-th iteration, and they are the same as the equations in the frequency-domain adaptive filter.,10) Therefore, the behavior of the MEFX LMS algorithm can be estimated at each frequency bin $\omega$. In other words, the convergence characteristics with the MEFX LMS algorithm in the time domain are approxi- mately determined by $N / 2+1$ independent frequency bin modes in the frequency domain.

At each frequency bin $\omega$, using the conventional method to evaluate the convergence characteristics with the LMS algorithm in the time domain, the convergence characteristics with the MEFX LMS algorithm are associated with the eigenvalues of the spectral matrix $\boldsymbol{R}(\omega),{ }^{3,4)}$ defined by

$$
\boldsymbol{R}(\omega)=E\left[\boldsymbol{U}^{\mathrm{H}}(n, \omega) \boldsymbol{U}(n, \omega)\right] .
$$

The size of the spectral matrix $\boldsymbol{R}(\omega)$ is $M K \times M K$ at each frequency bin $\omega$. The determinant of the spectral matrix $\boldsymbol{R}(\omega)$ can be expressed as the following equation in the frequency domain ${ }^{4)}$ :

$$
\begin{aligned}
|\boldsymbol{R}(\omega)|= & E\left[\left|X_{1}\right|^{2}(\omega)\right] E\left[\left|X_{2}\right|^{2}(\omega)\right] \\
& \cdots E\left[\left|X_{K}\right|^{2}(\omega)\right]\left|\boldsymbol{C}^{\mathrm{H}}(\omega) \boldsymbol{C}(\omega)\right|^{K},
\end{aligned}
$$

where $E\left[\left|X_{1}\right|^{2}\right], \cdots, E\left[\left|X_{K}\right|^{2}\right]$ are the input power spectra, and $C$ is the secondary path matrix

$$
\boldsymbol{C}=\left[\begin{array}{cccc}
C_{11} & C_{12} & \cdots & C_{1 M} \\
C_{21} & C_{22} & \cdots & C_{2 M} \\
\cdots \cdots \cdots \cdots \cdots \cdots \cdots & \cdots \cdots \cdots \cdots \\
\hdashline C_{L 1} & C_{L 2} & \cdots & C_{L M}
\end{array}\right],
$$

where element $C_{l m}$ is a transfer function from the $m$-th secondary source to the $l$-th error sensor.

\subsection{Effects of the Secondary Path Matrix $C$}

In order to study the effect of the matrix $C^{\mathrm{H}} \boldsymbol{C}$ in detail, it is assumed for simplicity that the reference signals are white noise with zero mean and variance $\sigma^{2}$. Then, the smallest eigenvalue of matrix $\boldsymbol{R}$ is influenced only by the smallest eigenvalue of matrix $\boldsymbol{C}^{\mathrm{H}} \boldsymbol{C}$, or the determinant of matrix $\boldsymbol{C}^{\mathrm{H}} \boldsymbol{C}$ at a frequency bin.

Therefore, we can evaluate the convergence speed at every frequency bin $\omega$ with the ratio $\rho(\omega)$ (see Appendix), defined by the following equation:

$$
\rho(\omega)=\frac{\max _{\omega}\left\{\left|\boldsymbol{C}^{\mathrm{H}}(\omega) \boldsymbol{C}(\omega)\right|\right\}}{\left|\boldsymbol{C}^{\mathrm{H}}(\omega) \boldsymbol{C}(\omega)\right|},
$$

where $\max _{\omega}\{\}$ denotes the maximum value over the whole frequency range concerned. Thus, we can evaluate the convergence speed approximately by using the ratio $\rho$, instead of the eigenvalues of the matrix $\boldsymbol{R}$ or $\boldsymbol{C}^{\mathrm{H}} \boldsymbol{C}$. The physical meaning of the matrix $\boldsymbol{C}^{\mathrm{H}} \boldsymbol{C}$ was discussed in a previous paper. ${ }^{4)}$ In a single channel ANC system, the matrix $C(\omega)$ is represented by $1 \times 1$ matrix, and the ratio $\rho(\omega)$ can 
be written as

$$
\rho(\omega)=\frac{\max _{\omega}\left\{|C(\omega)|^{2}\right\}}{|C(\omega)|^{2}},
$$

where $|C(\omega)|^{2}$ is the power gain of the transfer function.

It is found that if $\left|\boldsymbol{C}^{\mathrm{H}} \boldsymbol{C}\right|$ (or $|\boldsymbol{C}|^{2}$ ) is small, the convergence speed of the mean square error is slow, computation error is large, and the amount of cancellation is small. ${ }^{3,4)}$

\section{IMPROVEMENT OF CONVERGENCE CHARACTERISTICS}

By analysis in the frequency domain, the influence of the secondary paths on the convergence speed with the adaptive LMS algorithm is subject to the distribution of either the eigenvalues or the determinant of $\boldsymbol{C}^{\mathrm{H}} \boldsymbol{C}$. That is, if $\left|\boldsymbol{C}^{\mathrm{H}} \boldsymbol{C}\right|$ is relatively small at a certain frequency bin $\omega$, it results in a slow convergence speed, a large computation error and less sound cancellation. In other words, if $\left|\boldsymbol{C}^{\mathrm{H}} \boldsymbol{C}\right|$ shows a large dip at a frequency bin $\omega$, the convergence speed of the adaptive filter is slow at that frequency bin $\omega$, and the total convergence speed is also slow.

Since the transfer function of the secondary paths should be measured prior to the ANC processing, the influence of secondary paths on the convergence characteristics can be evaluated in advance. Thus, in this section, three methods are investigated in detail by which $\left|\boldsymbol{C}^{\mathrm{H}} \boldsymbol{C}\right|$ can be augmented large at some frequency bins where its value is small. In the next discussion, numerical simulations were carried out to demonstrate the theoretical results. All the simulations were performed in time domain, but their evaluation was carried out both in the time and frequency domains. For convenience, the experimental conditions were determined as follows: (1) Noise sources were uncorrelated white noise with zero mean and variance one. (2) The responses of the primary paths, $H$, and secondary paths $C$, were experimentally obtained at a $2 \mathrm{kHz}$ sampling frequency in a room with a reverberation time of approximately $0.5 \mathrm{~s}$. (3) The length of the impulse response of the primary paths and the secondary paths were 512 and 128, respectively. (4) The length of the adaptive filters and the length of the FFT were 512. (5) The initial value of the adaptive filter $\boldsymbol{w}(0)=\mathbf{0}$ in time domain, then $W_{0}(\omega)=0$ at each frequency bin $\omega$. (6) Assuming no external noise. (7) The learning curve, that is the mean square error (MSE) against number of iterations defined by Eq. (11), was evaluated through the simulation,

$$
\operatorname{MSE}[\mathrm{dB}]=-10 \log _{10} \frac{E\left[\sum e^{2}(n)\right]}{E\left[\sum d^{2}(n)\right]},
$$

where $E\left[\sum d^{2}(n)\right]$ is the average power of the desired signal due to the primary noises, and $E\left[\sum e^{2}(n)\right]$ is the average power of the residual at the error sensors.

\subsection{Position Adjustment}

To achieve a better convergence speed, in the case of a single secondary source and a single error sensor, a possible solution is to adjust their positions, and whether their position are good no not can be evaluated with the transfer function before performing the cancellation. In the case of multiple secondary sources and error sensors, it is found from section 2 that the suitability of their positions can be evaluated by observing $\left|\boldsymbol{C}^{\mathrm{H}} \boldsymbol{C}\right|$. A simulation was carried to performed the possibility of this method, using a CASE[2, 2, 2] ANC system. The determinants of $\left|\boldsymbol{C}^{\mathrm{H}} \boldsymbol{C}\right|$ are plotted as the solid line

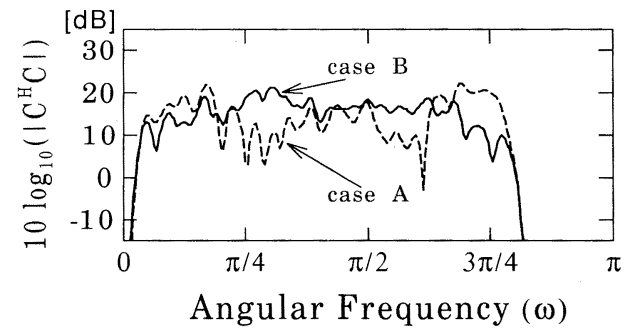

Fig. 2 The solid and dashed lines are the determinant $10 \log _{10}\left(\left|\boldsymbol{C}_{22}{ }^{\mathrm{H}} \boldsymbol{C}_{22}\right|\right)$ in case $\mathrm{A}$ and case $\mathrm{B}$, respectively.

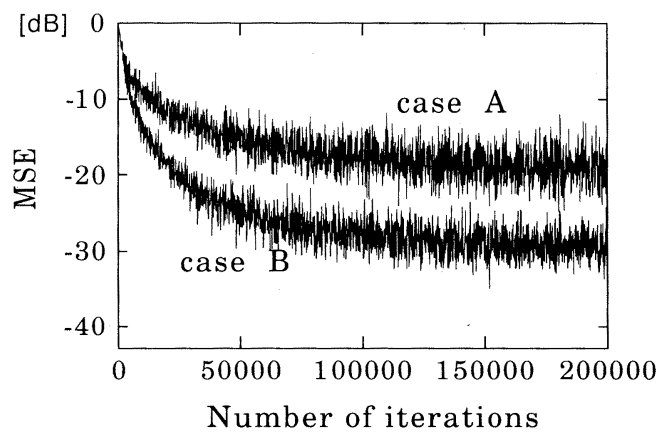

Fig. 3 Learning curves of the simulation with the MEFX algorithm using experimentally obtained secondary paths $C$. 


\section{G. CHEN et al.: IMPROVEMENT ANC SYSTEM CHARACTERISTICS}

(case A) and dashed line (case B) in Fig. 2. Case A (solid line) shows that the smallest value of $\left|\boldsymbol{C}^{\mathrm{H}} \boldsymbol{C}\right|$ is larger than that in case B (dashed line). Figure 3 shows the learning curves of case $\mathrm{A}$ and case $\mathrm{B}$. As expected, the convergence speed in case $\mathrm{A}$ is faster than that in case $B$, and the amount of cancellation in case $\mathrm{A}$ is larger than that in case $\mathrm{B}$. These simulations demonstrate the possibility of improving the convergence characteristics by adjusting the positions of secondary sources and error sensors appropriately. A higher convergence speed and a larger cancellation of noise are achieved if the difference of the determinant of the matrix $\left|\boldsymbol{C}^{\mathrm{H}} \boldsymbol{C}\right|$ is smaller. ${ }^{3)}$ However, this method does not always offer a smaller difference of $\left|\boldsymbol{C}^{\mathrm{H}} \boldsymbol{C}\right|$ over the whole frequency range.

\subsection{Increasing the Number of Secondary Sources}

Another possible solution is to increase the number of secondary sources, ${ }^{6)}$ where all of the transfer functions from the loudspeakers to sensors were known and remained unchanged. Here, it is described that this method makes some small values in $\left|\boldsymbol{C}^{\text {н }} \boldsymbol{C}\right|$ larger over the whole frequency range when there is no common zero in the transfer functions, and it works well in the case of ANC system using adaptive algorithms.

According to analysis in either the frequency or time domains, when $M \leq L$, either matrix $\boldsymbol{C}^{\mathrm{H}} \boldsymbol{C}$ or $\boldsymbol{R}$ is almost always positive definite. The adaptive filters have one and only one optimal solution and the error surface has a unique global minimum. The adaptive filters converge at this minimum value because of the adaptive algorithm. ${ }^{11,12)}$

When $\boldsymbol{M}>L$, either matrix $\boldsymbol{C}^{\mathrm{H}} \boldsymbol{C}$ or $\boldsymbol{R}$ is singular. In this case, these are indefinite optimal solutions of the adaptive filters, and there are indefinite smallest values of the error surface. ${ }^{13)}$ Thus, the classical method for analyzing the eigenvalues of matrix cannot be used to evaluate the convergence characteristics.

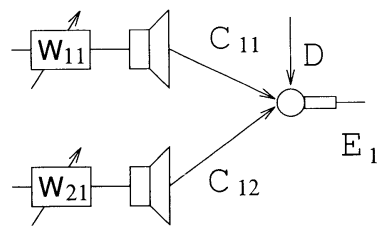

Fig. 4 Block diagram of a $\operatorname{CASE}[1,2,1]$ system.
Here, we will discuss the convergence characteristics with the adaptive algorithm when $M>L$, using a CASE $[1,2,1]$ system shown in Fig. 4.

In such CASE[1, 2, 1] ANC system, from Eqs. (4) and (5), the update equation in the frequency domain can be approximately written as

$$
\begin{aligned}
W_{11}(n+1, \omega)= & {\left[1-2 \mu^{\prime}\left|U_{11}(n, \omega)\right|^{2}\right] W_{11}(n, \omega) } \\
& -2 \mu^{\prime} U_{11}^{*}(n, \omega) D(n, \omega), \\
W_{21}(n+1, \omega)= & {\left[1-2 \mu^{\prime}\left|U_{12}(n, \omega)\right|^{2}\right] W_{21}(n, \omega) } \\
& -2 \mu^{\prime} U_{12}^{*}(n, \omega) D(n, \omega),
\end{aligned}
$$

where the asterisk denotes the complex conjugates, $U_{11}(n, \omega)=C_{11}(\omega) X(n, \omega)$ and

$U_{12}(n, \omega)=C_{12}(\omega) X(n, \omega)$.

From Eqs. (12) and (13), the update equation can be rewritten as

$$
\begin{aligned}
& \frac{W_{11}(n+1, \omega)-W_{11}(n, \omega)}{C_{11}^{*}(\omega)} \\
& =\frac{W_{21}(n+1, \omega)-W_{21}(n, \omega)}{C_{12}^{*}(\omega)} .
\end{aligned}
$$

This equation gives the convergence direction of the adaptive filter. That is, the convergence direction is the shortest distance from the initial value of the filter coefficients to the optimal set, as shown in Fig. 5. The convergence direction is different if the initial coefficients of the adaptive filters are different. If the initial value is 0 , the adaptive filters will converge at the minimum-norm solution, that is the residual power will be minimum.

The convergence speed is subject to the value $\lambda_{1}=$ $\left|C_{11}\right|^{2}+\left|C_{12}\right|^{2}$ in the computer simulation. If there is no common zero in the transfer functions of the two secondary paths, the following relation is valid :

$$
\frac{\max _{\omega}\left\{\left|C_{11}(\omega)\right|^{2}+\left|C_{12}(\omega)\right|^{2}\right\}}{\min _{\omega}\left\{\left|C_{11}(\omega)\right|^{2}+\left|C_{12}(\omega)\right|^{2}\right\}}<\frac{\max _{\omega}\left\{\left|C_{11}(\omega)\right|^{2}\right\}}{\min _{\omega}\left\{\left|C_{11}(\omega)\right|^{2}\right\}},
$$

or, equivalently,

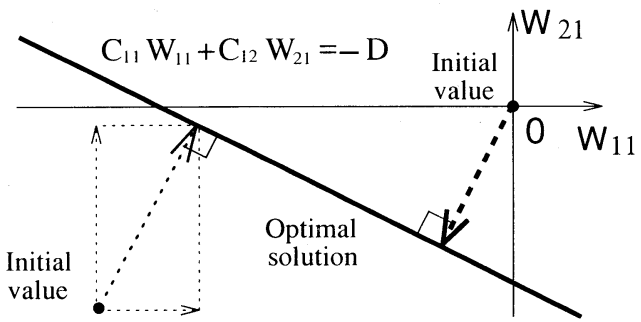

Fig. 5 The convergence direction of a CASE $[1$, $2,1]$ system. 


$$
\frac{\max _{\omega}\left\{\left|\boldsymbol{C}_{12}(\omega) \boldsymbol{C}_{12}{ }^{\mathrm{H}}(\omega)\right|\right\}}{\min _{\omega}\left\{\left|\boldsymbol{C}_{12}(\omega) \boldsymbol{C}_{12}{ }^{\mathrm{H}}(\omega)\right|\right\}}<\frac{\max _{\omega}\left\{\left|\boldsymbol{C}_{11}{ }^{\mathrm{H}}(\omega) \boldsymbol{C}_{11}(\omega)\right|\right\}}{\min _{\omega}\left\{\left|\boldsymbol{C}_{11}{ }^{\mathrm{H}}(\omega) \boldsymbol{C}_{11}(\omega)\right|\right\}} .
$$

When $M>L$, the convergence characteristics depend on the value $\boldsymbol{C} \boldsymbol{C}^{\mathrm{H}}$ instead of $\boldsymbol{C}^{\mathrm{H}} \boldsymbol{C}$, since $\boldsymbol{C}^{\mathrm{H}} \boldsymbol{C}=0$ where the nonzero eigenvalues of the matrix $\boldsymbol{C}^{\mathrm{H}} \boldsymbol{C}$ or $\boldsymbol{C} \boldsymbol{C}^{\mathrm{H}}$ are the same. The value $\boldsymbol{C} \boldsymbol{C}^{\mathrm{H}}$ over the whole frequency range will become flatter by increasing the number of secondary sources when there is no common zero in the transfer functions. Then, the ratio $\rho$ defined by Eq. (9) will be smaller, and the convergence speed of the adaptive algorithm becomes faster simultaneously.

For the CASE[2,2,2] and the CASE[2,3,2] systems, the value of $\left|\boldsymbol{C}_{22}{ }^{\mathrm{H}} \boldsymbol{C}_{22}\right|$ (or $\left|\boldsymbol{C}_{22} \boldsymbol{C}_{22}{ }^{\mathrm{H}}\right|$ ) and $\left|\boldsymbol{C}_{23} \boldsymbol{C}_{23}{ }^{\mathrm{H}}\right|$ are plotted in Fig. 6. The value of $\left|\boldsymbol{C}_{23} \boldsymbol{C}_{23}{ }^{\mathrm{H}}\right|$ over the whole frequency range is flatter in comparison with that of $\left|\boldsymbol{C}_{22}{ }^{\mathrm{H}} \boldsymbol{C}_{22}\right|$. Figure 7 shows the learning curves of a simulation of $\operatorname{CASE}[2,2,2]$ and CASE[2, 3, 2] ANC systems. As expected, the convergence speed of the CASE[2,3,2] system is

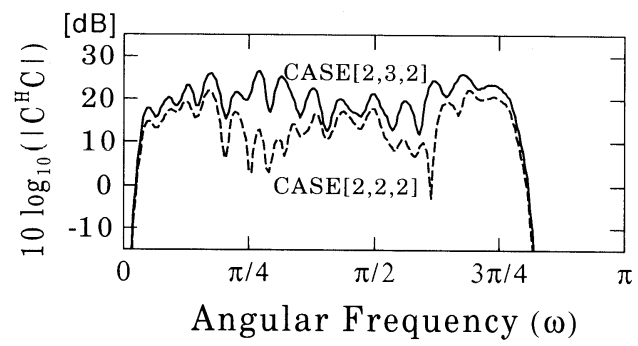

Fig. 6 Determinants of matrices $\boldsymbol{C}^{\mathrm{H}} \boldsymbol{C}$ and $C \boldsymbol{C}^{\mathrm{H}}$. The dashed line is $10 \log _{10}\left(\left|\boldsymbol{C}_{22}{ }^{\mathrm{H}} \boldsymbol{C}_{22}\right|\right)$, and the solid line is $10 \log _{10}\left(\left|\boldsymbol{C}_{23} \boldsymbol{C}_{23}{ }^{\mathrm{H}}\right|\right)$.

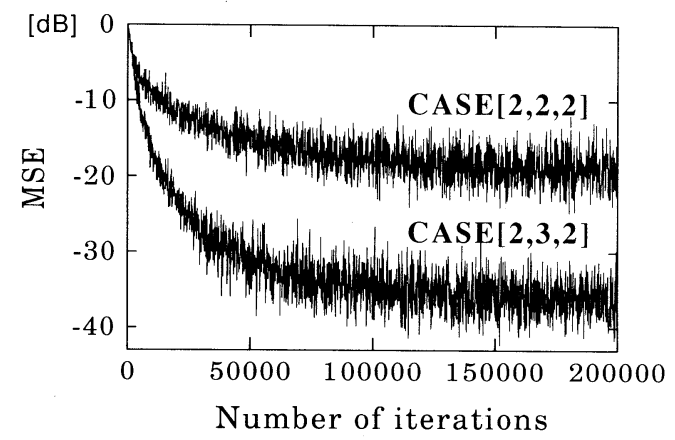

Fig. 7 Simulated learning curves in CASE[2, 2, 2] and $\operatorname{CASE}[2,3,2]$ systems in the time domain. faster than that of the $\operatorname{CASE}[2,2,2]$ system, and the cancellation of sound is larger. Further, this simulation shows that the convergence characteristics with the MEFX algorithm will be increased by adding a secondary source when there is no common zero in the transfer functions.

This result is the same as in the MINT method ${ }^{6,14)}$ which avoids the zero point (dip). The MINT method was proposed for realizing an exact inverse filter for acoustic impulse responses in a room. Here, we showed that the MEFX LMS algorithm in an ANC system with the MINT method offers.

\subsection{The Use of an Inverse Filter}

It is clear that if $\left|\boldsymbol{C}^{\mathrm{H}} \boldsymbol{C}\right|$ or $|\boldsymbol{C}|$ is relatively small at a certain frequency bin $\omega$, it will result in a slower convergence speed, a larger computation error and less cancellation at that bin. To reduce the influence of the dips in the transfer function of the secondary path, a use of inverse filter of the transfer function was proposed. ${ }^{7)}$ In this case, however, the formulation of the adaptive algorithm has not been performed. Therefore, in this section we first formulate the algorithm and discuss how to reduce the influence of the dips in the transfer function of the secondary path using a CASE $[1,1,1]$ system.

From Eq. (5), the update equation of the filtered-x LMS algorithm in the frequency domain can be written as

$$
\begin{aligned}
& W(n+1, \omega) \\
& \quad=W(n, \omega)-2 \mu^{\prime} C^{*}(\omega) X^{*}(n, \omega) E(n, \omega) .
\end{aligned}
$$

In the time domain, the step-size parameter $\mu^{\prime}$ is a constant, but this $\mu^{\prime}$ may change according to the frequency bin $\omega$ in the frequency domain as

$$
\begin{aligned}
& W(n+1, \omega) \\
& \quad=W(n, \omega)-2 \mu^{\prime}(\omega) C^{*}(\omega) X^{*}(n, \omega) E(n, \omega) .
\end{aligned}
$$

To remove the influence of the transfer function, the step-size parameter is defined by

$$
\mu^{\prime}(\omega)=\frac{\mu^{\prime \prime}}{C^{*}(\omega)}=\mu^{\prime \prime} C^{*-1}(\omega),
$$

where $\mu^{\prime \prime}$ is a constant. By substituting Eq. (19) in the update equation, Eq. (18) can be rewritten as,

$$
\begin{aligned}
& W(n+1, \omega) \\
& \quad=W(n, \omega)-2 \mu^{\prime \prime} C^{*-1}(\omega) C^{*}(\omega) X^{*}(n, \omega) E(n, \omega) \\
& \quad=W(n, \omega)-2 \mu^{\prime \prime} X^{*}(n, \omega) E(n, \omega) .
\end{aligned}
$$

It can be seen that the influence of the transfer function $C(\omega)$ in the filtered-x LMS algorithm 


\section{G. CHEN et al.: IMPROVEMENT ANC SYSTEM CHARACTERISTICS}

disappears. In the time domain, Eq. (20) can be rewritten as

$$
\boldsymbol{w}(n+1)=\boldsymbol{w}(n)-2 \mu^{\prime \prime} c^{-1} \boldsymbol{u}(n) e(n) .
$$

An ANC system, which theoretically improves the convergence speed by introducing an inverse-c filter, ${ }^{7)}$ is shown in Fig. 8. In this system, the filtered reference signal can be written as

$$
f=\widehat{c^{-1} \otimes c} \text {, }
$$

where $\otimes$ is the convolution operator and ${ }^{\wedge}$ means that the response $f$ is realized by an FIR filter. 'In the theoretical analysis, $f$ can be represented by a simple time delay. A simulation was executed by using a transfer function as the secondary path $C$, which was experimentally obtained in a $\operatorname{CASE}[1,1$, 1] ANC system. As shown in Fig. 9, there are some dips (small values) in the power gain of the transfer function $C$ throughout the frequency range. Also, using an inversed-c filter increased the power gain of the transfer function of $f=c^{-1} \otimes c$. Thus, a faster convergence speed is expected with the inversed-c filter. Figure 10 shows the power spectra (12 runs) of the residual noise at the error sensor during adaptation at 25,000 iterations with filtered-x LMS and inversed-c LMS algorithms. Figure 11 shows

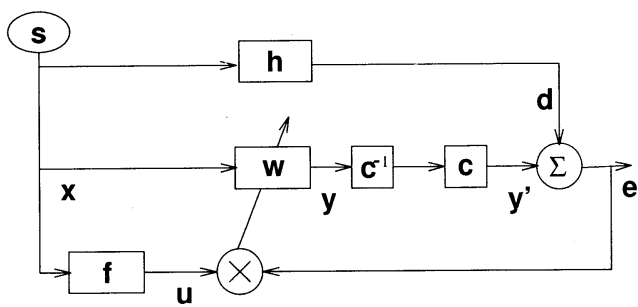

Fig. 8 Diagram of a single channel ANC system implemented with the inversed-c algorithm.

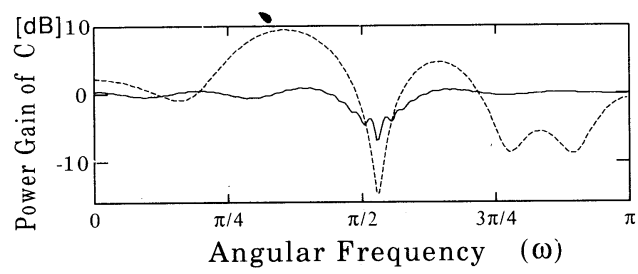

Fig. 9 Power gain of the secondary path $C$ (dashed line: $10 \log _{10}\left(|C|^{2}\right)$ ) measured in a room, and the power gain of $f=c^{-1} \otimes c$ (solid line: $10 \log _{10}\left(\left|C^{-1} C\right|^{2}\right)$ ) by introducing an inversed-c filter. the learning curves of the simulation with the filtered-x LMS algorithm, using the secondary path $C$, and with the inversed-c LMS algorithm, implemented by introducing the inversed-c filter. These simulations show that the inversed-c LMS algorithm offers a faster convergence speed and a larger cancellation of sound than the conventional filtered-x LMS algorithm.

When the inverse filter $c^{-1}$ cannot be completely calculated, the response $f$ should be realized by using an FIR filter. In most cases, the FIR filter of $f$ is shorter than the FIR filter of $\hat{c}$, which substitutes for the response of the secondary path $C$. As shown in Fig. 11 about $30 \mathrm{~dB}$ of noise attenuation was achieved with this method for the secondary path $C$ measured in a room with reverberation time of $0.5 \mathrm{~s}$. This shows that the method using the inverse filter $c^{-1}$ may be effective in practice.

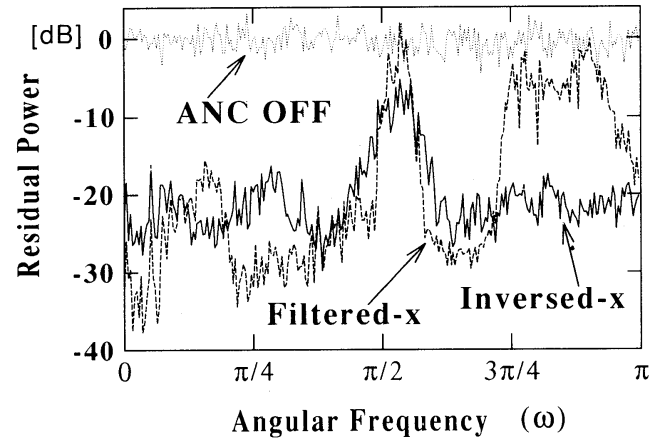

Fig. 10 Power spectrum (average of 12 times) of the residual noise at the error sensor during adaptation (iterations : 25,000 times).

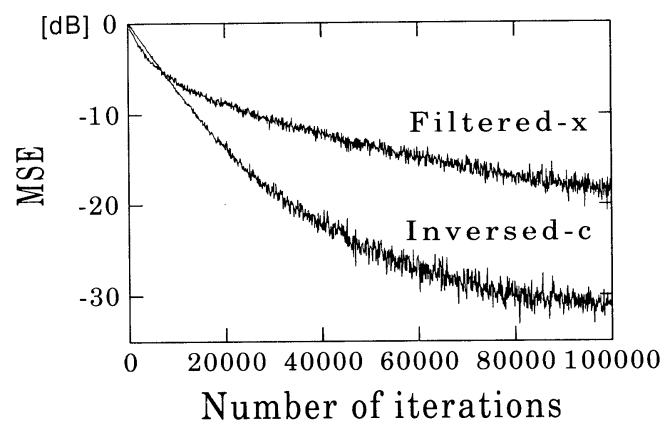

Fig. 11 Learning curves of the simulation by the filtered-x algorithm, using an experimentally estimated secondary path $C$, and by the inversed-c algorithm using an inversed-c filter. 


\section{CONCLUSIONS}

The convergence characteristics of the filtered-x LMS and MEFX LMS algorithms in the time domain could be analyzed in the frequency domain with fewer computations and with better understanding of the physical meaning than in the time domain. That is, the influence of the secondary paths on the convergence speed in the adaptive LMS algorithm depends on the determinant of the matrix $\boldsymbol{C}^{\mathrm{H}} \boldsymbol{C}$ or $\boldsymbol{C} \boldsymbol{C}^{\mathrm{H}}$. At a certain frequency bin, where the value of the determinant is relatively small, this results in a slow convergence speed, a large computation error and less cancellation of noise at the frequency bin.

This paper discussed on three solutions to achieve the faster convergence speed and larger noise cancellation, by increasing the value of $\left|\boldsymbol{C}^{\mathrm{H}} \boldsymbol{C}\right|$ at frequencies where the $\left|\boldsymbol{C}^{\mathrm{H}} \boldsymbol{C}\right|$ is small. For adjusting the positions of secondary sources and error sensors, an index $\left|\boldsymbol{C}^{\mathrm{H}} \boldsymbol{C}\right|$ was proposed to evaluate whether their positions of multiple secondary sources and error sensors are suitable or not. For the second method in which the number of secondary sources was increased, the LMS adaptive algorithm was shown to be applicable to it through simulation. For the third method in which the inverse filters of the secondary paths, about $30 \mathrm{~dB}$ of noise attenuation could be achieved by the simulation using the actual transfer function $C$ measured in a room with reverberation time of $0.5 \mathrm{~s}$. This result shows that the method using an inverse filter may be actually useful.

The theoretical results *were verified through computer simulations with the time-domain filtered-x LMS and MEFX LMS algorithms and the convergence speed was evaluated in both the time and frequency domains.

\section{ACKNOWLEDGEMENT}

The authors would like to thank Dr. Yôiti Suzuki of Tohoku University for his useful comments.

\section{REFERENCES}

1) B. Widrow and S. D. Stearns, Adaptive Signal Processing (Prentice-Hall Inc., Englewood Cliffs, 1985), pp. 101-114, 288-292.

2) S. J. Elliott, I. M. Stothers, and P. A. Nelson, "A multiple error LMS algorithm and its application to active control of sound and vibration," IEEE Trans. Acoust. Speech Signal Proc. ASSP-35, 1423-1434
(1987).

3) G. Chen, M. Abe, and T. Sone, "Evaluation of the convergence characteristic of the filtered-x LMS algorithm in frequency domain," J. Acoust. Soc. Jpn. (E) 16, 331-340 (1995).

4) G. Chen, M. Abe, and T. Sone, "Effects of multiple secondary paths on convergence properties in active noise control systems with LMS algorithm," J. Sound Vib. 195, 217-228 (1996).

5) J. D. Stell and R. J. Bernhard, "Active control of sound in acoustic waveguides, part II : considerations for implementation in ducts," J. Sound Vib. 173, 197 215 (1993).

6) M. Miyoshi and Y. Kaneda, "Inverse filtering of room acoustics," IEEE Trans. Acoust. Speech Signal Process. ASSP-36, 145-152 (1988).

7) N. Saito, H. Sano, and H. Sawada, "The method of noise cancellation," Japan Patent No. 6-27972 (1994) (in Japanese).

8) S. J. Elliott and P. A. Nelson, "Active noise control," IEEE Signal Process. Mag., 12-35 (1993).

9) S. S. Narayan and A.M. Peterson, "Frequency domain least-mean-square algorithm," Proc. IEEE 69, 124-126 (1981).

10) M. Dentino, J. McCool, and B. Widrow, "Adaptive filtering in the frequency domain," Proc. IEEE 66, 1658-1659 (1978).

11) S. Haykin, Introduction to Adaptive Filters (Macmillan, New York, 1984).

12) H. Hamada, "Fundamentals of adaptive digital filter (Part 2),” J. Acoust. Soc. Jpn. (J) 45, 731-738 (1989) (in Japanese).

13) G. Chen, M. Abe, and T. Sone, "Improvement of the convergence properties of the ANC system based on analysis in the frequency domain," Proc. ACTIVE 95, 1013-1024 (1995).

14) M. Miyoshi and Y. Kaneda, "Active noise control using inverse filtering of a sound field in a room," J. Acoust. Soc. Jpn. (J) 46, 3-10 (1990) (in Japanese).

15) G. Strang, Linear Algebra and Its Applications (Academic Press, New York, 1976).

\section{APPENDIX A DERIVATION OF EQ. (9)}

In the frequency domain analysis, the convergence characteristics of the MEFX LMS algorithm are associated with the eigenvalues of the spectral matrix $\boldsymbol{R}(\omega)$ defined by Eq. (6) at each frequency bin $\omega^{3,4)}$

As well as the analysis in the time domain, the upper limit of $\mu^{\prime}$ and the longest time constant $\tau_{\max }(\omega)$ at a frequency bin $\omega$ can be obtained as

$$
\begin{gathered}
0<\mu^{\prime}<\frac{1}{\lambda_{\max }(\omega)}, \\
\tau_{\max }(\omega)>\frac{1}{2 \mu^{\prime} \lambda_{\min }(\omega)},
\end{gathered}
$$

where $\lambda_{\max }(\omega)$ and $\lambda_{\min }(\omega)$ are the largest and small- 


\section{G. CHEN et al.: IMPROVEMENT ANC SYSTEM CHARACTERISTICS}

est eigenvalues of $\boldsymbol{R}(\omega)$ at a frequency bin $\omega$.

Over the whole frequency range (for all the frequency bin $\omega$ ) concerned, the upper limit of the $\mu^{\prime}$ is given by

$$
0<\mu^{\prime}<\frac{1}{\max _{\omega}\left\{\lambda_{\max }(\omega)\right\}},
$$

where $\max _{\omega}\{\quad\}$ denotes the maximum value over the whole frequency range.

Thus, substituting Eq. (A.3) into Eq. (A.2), the longest time constant is as

$$
\tau_{\max }(\omega)>\frac{\max _{\omega}\left\{\lambda_{\max }(\omega)\right\}}{2 \lambda_{\min }(\omega)} .
$$

Since the determinant of the matrix is equal to the product of its eigenvalues, ${ }^{15)}$ the eigenvalues of matrix $\boldsymbol{R}$ are influenced by the eigenvalues of matrix $\boldsymbol{C}^{\mathrm{H}} \boldsymbol{C}$. In general, if the determinant of matrix $\boldsymbol{C}^{\mathrm{H}} \boldsymbol{C}$ is smaller, the smallest eigenvalue of matrix $\boldsymbol{C}^{\mathrm{H}} \boldsymbol{C}$ is smaller, the smallest eigenvalue of matrix $\boldsymbol{R}$ is also smaller, and the convergence speed is slower. Therefore, we can evaluate the convergence speed at every frequency bin $\omega$ by the ratio $\rho(\omega)$ defined by the following equation instead of Eq. (A.4) :

$$
\rho(\omega)=\frac{\max _{\omega}\left\{\left|\boldsymbol{C}^{\mathrm{H}}(\omega) \boldsymbol{C}(\omega)\right|\right\}}{\left|\boldsymbol{C}^{\mathrm{H}}(\omega) \boldsymbol{C}(\omega)\right|} .
$$

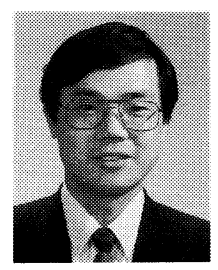

Guoyue Chen was born in Shanghai, P. R. China on May 15, 1961, received his B.S. degree from East China Normal University, Shanghai, China in 1983. He was a research associate at Department of Computer Science, East China Normal University from 1983 to 1989. He received the M.S. and Ph.D. degree from Tohoku University, Japan in 1993 and 1996, respectively. He is now a research associate at Computer Center, Tohoku University of Japan. His research interests include digital signal processing and image processing. Dr. Chen is a member of the Acoustical Society of Japan and the Institute of Electronics, Information and Communication Engineers of Japan.

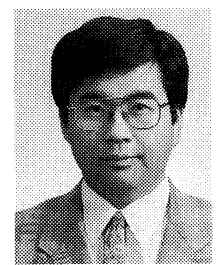

Masoto Abe was born in Miyagi, Japan on May 3, 1952. He received the B.E., M.S. and Ph.D. degrees from Tohoku University, Sendai, Japan in 1976, 1978 and 1981, respectively. From 1981 to 1989 he was at Research Center for Applied Information Sciences, Tohoku University as a research associate. From 1989 to 1996 he was an associate professor at Computer Center, Tohoku University. He is now a professor of Iwate University, Japan. His research interests digital signal processing for acoustics and computer architecture. Dr. Abe is a member of the IEEE, the Acoustical Soc. of America, the Acoustical Soc. of Japan, the Institute of Noise Control Engineering of Japan and Information Processing Society of Japan.

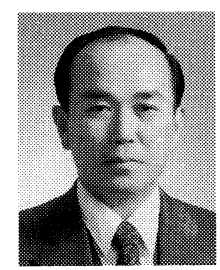

Toshio Sone was born on 14 May 1935. A graduate in electrical engineering at Tohoku University, Japan in 1958. Sone did his graduate work at the same university, where he was awarded his Ph.D. in electrical and communication engineering in 1963. He is now a professor of the Research Institute of Electrical Communication, Tohoku University. He has been engaged in researches on psychological acoustics, electroacoustics, room acoustics and noise control over thirty years. From 1993 to 1995 he was President of the Acoustical Society of Japan. 